\title{
EXTREMELY WIDE TUNING RANGE OF MECHANICAL OSCILLATION OF A SILICON WAVEGUIDE DRIVEN BY OPTICAL GRADIENT FORCE
}

\author{
S. Tallur ${ }^{l, 2 *}$, and S.A. Bhave \\ ${ }^{1}$ Cornell University, Ithaca, USA, ${ }^{2}$ Analog Devices Inc., Wilmington, USA, \\ ${ }^{3}$ Purdue University, West Lafayette, USA
}

\begin{abstract}
MEMS oscillators are well-suited for filter and timing applications owing to high resonator quality factors. Active compensation of frequency variation in conventional commercial MEMS oscillators is limited by their tuning range, which is typically $<1,000 \mathrm{ppm}$. This paper demonstrates an extremely wide tuning range for oscillations of a silicon clamped-clamped beam via the optical spring effect. Frequency tuning from $3.1 \mathrm{MHz}$ to $19.4 \mathrm{MHz}$ is achieved by varying the intra-cavity power, corresponding to an optical-tuning coefficient of $1312.5 \mathrm{ppm} / \mu \mathrm{W}$. To the best of our knowledge, this is the largest reported tuning range in literature for MEMS oscillators.
\end{abstract}

\section{INTRODUCTION}

MEMS resonators are attractive for oscillator design on account of their extraordinary small size, high level of integration, low cost and high volume manufacturing capability. MEMS oscillators can be integrated either on the CMOS die or as two-chip solution using a separate CMOS die for interface electronics in a single package [1]. Typically high quality factors of MEMS resonators also enable superior noise performance and frequency stability compared to electrical oscillators. MEMS oscillators thus fill the gap between high-performance, non-CMOS compatible technologies on the one hand, and low-performance CMOS compatible technologies on the other.

Frequency stability is one of the most important parameters of an oscillator. Since the output of an oscillator is the frequency of oscillation, it is possible to use the frequency measurement in an active feedback loop to counter the mechanism that causes the shift in frequency. The frequency variation of MEMS oscillators is typically dominated by temperature fluctuations in the device. Compared to quartz, silicon resonators have much larger temperature coefficient of frequency (TCF) [2]. To overcome the linear TCF of MEMS resonators, recent advances $[3,4]$ have relied upon using oxide plugs to reduce the TCF magnitude to sub$25 \mathrm{ppm}$ and a quadratic temperature dependence of frequency. Achieving even lower TCF requires ovenization [5]. For commercial oscillators, tuning ranges on orders of few hundred ppm have been demonstrated [2, 4]. For voltage controlled crystal oscillators (VCXO), typical tuning coefficients are on the order of $\sim 100 \mathrm{ppm} / \mathrm{V}$. To counter frequency variation due to manufacturing imperfections and package stresses, and long term frequency drift due to aging, having a method to achieve larger tuning range without significant added power consumption and die-space is desirable.

Contrary to the small tuning range achieved by electrostatic spring softening, the optical spring effect has been leveraged to achieve significantly larger tuning ranges in opto-mechanical resonators $[6,7]$. In this paper, we demonstrate an all-optically transduced silicon opto-mechanical oscillator, and leverage the optical spring effect to show large frequency tuning range of $525 \%$ for an $\mathrm{n}=1$ in-plane bending mode of a clamped-clamped silicon beam from $3.1 \mathrm{MHz}$ to $19.4 \mathrm{MHz}$. The following section describes the theory of operation for the oscillator and the optical spring effect. The subsequent sections detail the device design, fabrication and experimental results.

\section{THEORY OF OPERATION}

\section{Optical gradient force driven mechanical oscillations}

Our group has previously demonstrated and explained the phenomenon of optical radiation pressure driven oscillations in an opto-mechanical resonator [8]. Alternatively, optical intensity gradients found in the near-field of guided wave nanostructures can be harnessed to create large gradient-like optical forces [9]. In contrast to the radiation pressure force, the gradient force depends upon the transverse evanescent-field coupling between adjacent cavity elements. One example of such a system that will be employed in this paper is a suspended optical waveguide coupled to an opto-mechanical cavity resonator. When the light wavelength is chosen such that it is detuned to an optical resonance of the resonator, the light intensity inside the cavity is enhanced due to its large optical quality factor. Thus if $\mathrm{P}_{\text {in }}$ represents the input laser power, and $\mathrm{Q}_{\mathrm{opt}}$ is the optical quality factor, the intra-cavity power is denoted by $\mathrm{P}_{\text {cavity }}=\mathrm{Q}_{\text {opt }} \mathrm{P}_{\text {in }}$. This results in a large field gradient between the light field in the cavity and light field in the waveguide $\left(\mathrm{P}_{\text {in }}\right)$ over a small gap.

The optical gradient force acting on the resonator results in an optical anti-damping force acting on the waveguide and the resonator. The anti-damping rate $\Gamma_{\mathrm{opt}}$ is proportional to the circulating optical power and the detuning of the laser light from the cavity resonance [10]. By adjusting both the power and the detuning, the anti-damping rate can be increased to overcome the intrinsic mechanical damping rate $\left(b=\Omega_{\mathrm{m}} / 2 \mathrm{Q}_{\text {mech }}\right.$, where $\Omega_{\mathrm{m}}$ and $Q_{\text {mech }}$ denote the mechanical resonance frequency and quality factor respectively) in the resonator or the waveguide. This results in self-sustained mechanical oscillations of the device.

\section{Optical spring effect}

According to the derivation in [9], the mechanical spring constant of the cavity (k') depends on the input laser power $\left(\mathrm{P}_{\text {in }}\right)$ as follows:

$$
k^{\prime}=k_{0}\left(1+\eta_{o s} P_{i n}\right)
$$

where $\mathrm{k}_{0}$ is the intrinsic mechanical spring constant and $\eta_{\mathrm{os}}$ is the optical spring coefficient. The optical spring coefficient varies with the detuning as follows:

$$
\eta_{o s} \propto d\left(Q_{o p t}\right)^{2} /\left[\Omega_{0}\left(d^{2}+0.25\right)\right]^{2}, d=\left(\lambda_{0}-\lambda_{\text {in }}\right) / \Delta \lambda_{L W}
$$

In the equation above, $\mathrm{d}$ denotes a normalized representation of the detuning that is in turn dependent on the input laser wavelength $\left(\lambda_{\text {in }}\right)$, the optical cavity resonant wavelength $\left(\lambda_{0}\right)$ and the line-width of the cavity resonance $\left(\Delta \lambda_{\mathrm{LW}}\right)$. The spring coefficient is directly proportional to the optical quality factor (Qopt) and inversely proportional to the intrinsic mechanical resonance frequency, suggesting that the largest tunability is noticed for low frequency mechanical modes excited with a high quality factor resonance. Oscillations occur only when the laser is blue detuned with respect to the cavity $(d>0)$. The optical spring coefficient is thus always positive (spring stiffening), and has the highest magnitude at $\mathrm{d} \approx 0.3$. Operating at this detuning results in the largest deviation from the natural mechanical resonance 
frequency for the oscillator. As the laser wavelength is increased and swept into resonance, the normalized detuning transitions from 1 to 0 , and the resulting mechanical resonance shifts from a rising trend to a falling trend, crossing a local maximum at $\mathrm{d} \approx 0.3$.

\section{DESIGN AND FABRICATION}

As noted in equation (2), the largest tuning range is observed for low frequency mechanical modes. This demonstration uses a silicon opto-mechanical resonator coupled to a suspended silicon waveguide. Since the micro-ring resonator is designed for higher frequency modes (radial breathing mode at $175 \mathrm{MHz}$ and compound radial mode at $1.1 \mathrm{GHz}$ ) [11], for this experiment we focus on the waveguide modes, which are at much lower frequencies (fundamental in-plane bending mode at $3.4 \mathrm{MHz}$ ). The device comprises of a silicon waveguide of length $30 \mu \mathrm{m}$ and width $400 \mathrm{~nm}$. The waveguide is coupled to a silicon opto-mechanical resonator. Each individual ring in the coupled resonator has a width of $3.8 \mu \mathrm{m}$. The device thickness is $220 \mathrm{~nm}$. The waveguideresonator gap is $<100 \mathrm{~nm}$. The fabrication process flow was described in detail in earlier work on multi-GHz opto-mechanical oscillations of the coupled ring resonator [11].

Fabricating the device involves a five mask process flow on a custom silicon-on-insulator (SOI) wafer (undoped $250 \mathrm{~nm}$ device layer for low optical loss and $3 \mu \mathrm{m}$ thick buried oxide for optical isolation from the silicon substrate). The top silicon is thermally oxidized to obtain a thin oxide hard mask layer of thickness $60 \mathrm{~nm}$ atop a $220 \mathrm{~nm}$ thick silicon device layer. The devices are defined in the oxide mask using electron beam lithography and a $\mathrm{CHF}_{3} / \mathrm{O}_{2}$ based reactive ion etcher. The pattern is then transferred into the silicon device layer using a chlorine based reactive ion etch. The mechanical resonator, the electrical routing beams and the bondpads are doped via boron ion implantation using a second photolithography mask to protect the optical section of the device. A third mask is then used to deposit metal over the bond pads for electrical contact using lift-off lithography. The metal stack used for the bond pads comprises of $25 \mathrm{~nm}$ Nickel, $25 \mathrm{~nm}$ Titanium and $50 \mathrm{~nm}$ Platinum, respectively in the order of deposition. For the experiment described in this paper, the second and third masks are not necessary. A fourth mask is used to define release window for the waveguide and opto-mechanical resonator via photolithography, followed by a timed release etch in buffered oxide etchant to undercut the devices. The samples are then dried using a critical point dryer to prevent stiction. Fig. 1 shows a scanning electron micrograph (SEM) of the fabricated device.

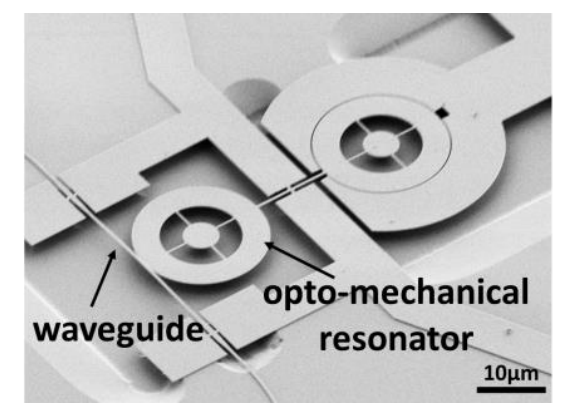

Figure 1: Scanning electron micrograph (SEM) of the device used for the experiments described in this manuscript.

\section{EXPERIMENTAL RESULTS}

Optical characterization

To characterize the optical transmission spectrum of the resonator, light from a continuous wave (CW) Santec TSL-510 tunable diode laser is coupled into the on-chip waveguide through grating couplers and the transmitted power is measured using an optical power meter. The wavelength of the laser is swept to identify optical resonances of the device. Fig. 2 shows an optical transmission spectrum of such a device. At low laser power $(0 \mathrm{Bm})$, the optical resonances are clearly seen as dips in the optical transmission spectrum. At higher laser power $(15 \mathrm{dBm})$, the thermal nonlinearity of the optical resonator combined with the mechanical oscillations of the waveguide distort the optical transmission spectrum.

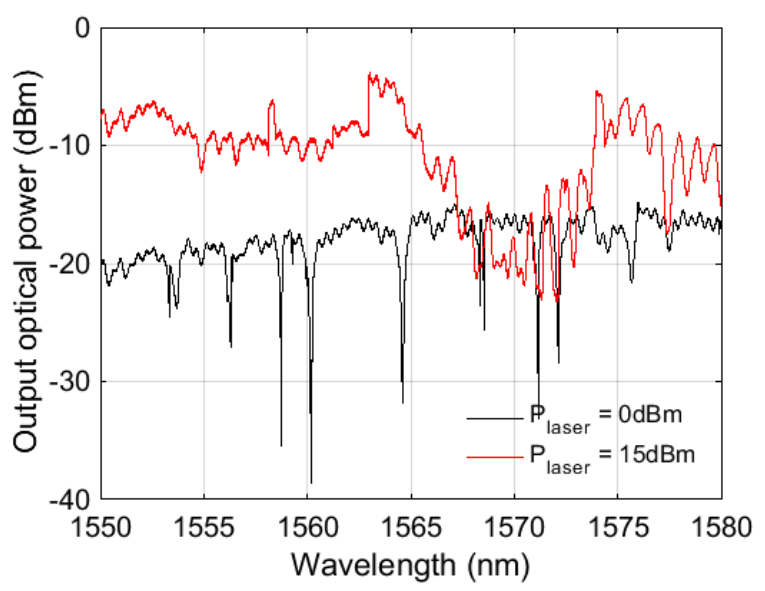

Figure 2: Comparison of optical output transmission spectra at $0 \mathrm{dBm}$ and $15 \mathrm{dBm}$ input laser power. Oscillations set in as the laser is swept beyond $1563 \mathrm{~nm}$ when operating with $15 \mathrm{dBm}$ power, manifesting as a distorted optical transmission spectrum.

\section{Mechanical oscillation}

To probe the mechanical mode of the waveguide, the transmitted light is passed to a Newfocus 1647 Avalanche Photodiode with gain setting of $6,000 \mathrm{~V} / \mathrm{W}$. The motion of the waveguide and the opto-mechanical ring resonator leads to phase modulation of the intra-cavity light, which is converted into amplitude modulation via shaping by the optical resonator transfer function [11]. The ring resonator is designed such that it does not have mechanical modes in the vicinity of the resonance frequency of the fundamental in-plane and out-of-plane bending modes of the waveguide. The RF signal is analyzed on an Agilent 5052B signal analyzer. Fig. 3 shows a sketch of the experimental setup.

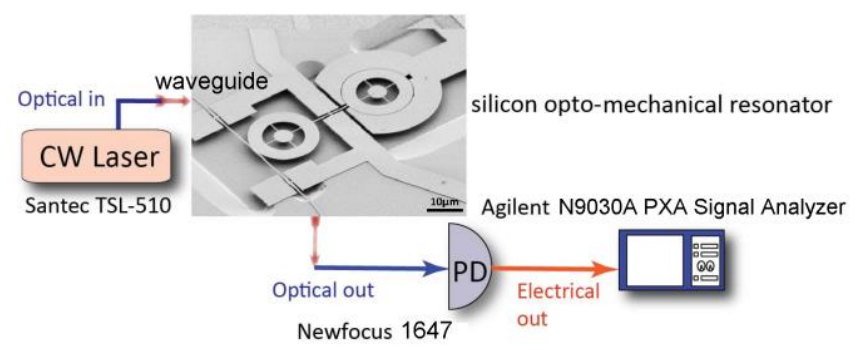

Figure 3. Experimental setup used to study opto-mechanical oscillation of the silicon waveguide.

As the laser wavelength is swept into an optical resonance of the cavity, the intra-cavity optical power in the resonator builds up. When the built up power is sufficiently high such that the antidamping introduced by the optical gradient force overcomes the intrinsic mechanical damping in the silicon waveguide, mechanical 
oscillation of the waveguide ensues. The optical power threshold required to launch oscillations is minimized when the detuning of the laser frequency $\left(\omega_{\text {in }}\right)$ from the cavity resonance $\left(\omega_{0}\right)$ is equal to the mechanical resonance frequency $\left(\Omega_{\mathrm{m}}\right)$ i.e. when $\omega_{\mathrm{in}}-\omega_{0}=\Omega_{\mathrm{m}}$. Fig. 4 below shows a comparison of the spectrum of the photodetector output for sub-threshold and above threshold operation. The Brownian noise peak of the $n=3$ in-plane bending mode of the waveguide is seen in the spectrum for the laser wavelength corresponding to sub-threshold operation. The tethers supporting the waveguide act as the anchors for the clamped-clamped beam. These are designed as a multi-mode interference (MMI) waveguide arm to minimize optical losses at the tether, and are not optimized for anchor losses of the mechanical mode. As such, the $n=3$ mode has very low mechanical quality factor of 250 . The spurs seen in the spectra are on account of the piezo controllers on the mirrors of the tunable laser.

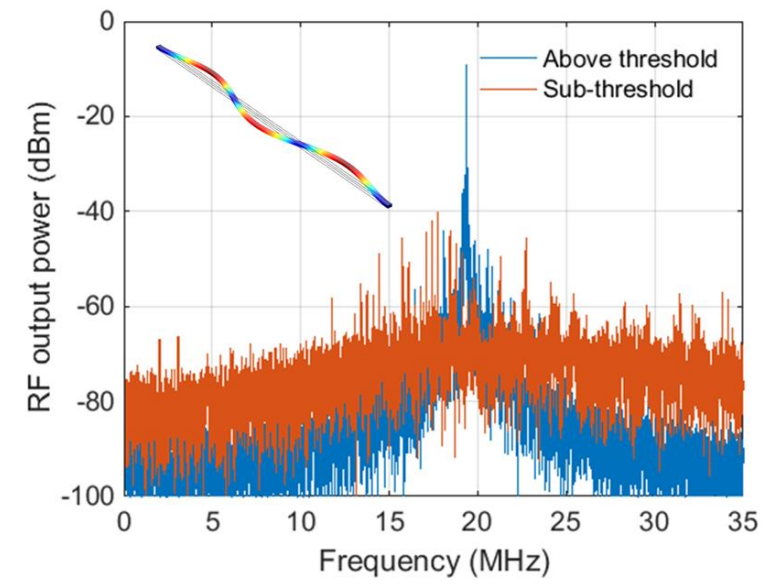

Figure 4. Comparison of Brownian noise spectrum of the $n=3$ inplane mode of vibration of the silicon waveguide (inset: FEM simulation) to onset of oscillations ushered in by detuning the wavelength to above-threshold operation.

\section{Frequency tuning of mechanical oscillation}

As described in the 'Theory of Operation' section above, the optical spring constant is dependent on the detuning of the laser to the optical resonance of the cavity. In our experiment, we manually sweep the laser wavelength from $1573 \mathrm{~nm}$ to $1577.5 \mathrm{~nm}$ in steps of $0.02 \mathrm{~nm}$, thereby scanning across multiple optical resonances of the cavity shown in Fig. 2. The input laser power is maintained at $14 \mathrm{dBm}$. When the laser wavelength is swept, the coupling changes from one optical resonance to another. Thereby the optical spring coefficient varies and passes through the local maxima for each resonance, as seen in the rising trend in oscillation frequency followed by a falling trend as seen in Fig. 5. The $n=1$ in-plane bending mode is thus tuned from $3.1 \mathrm{MHz}$ (natural resonance frequency at $\mathrm{d}=0$ ) to $19.4 \mathrm{MHz}$, corresponding to a $525 \%$ tuning range.

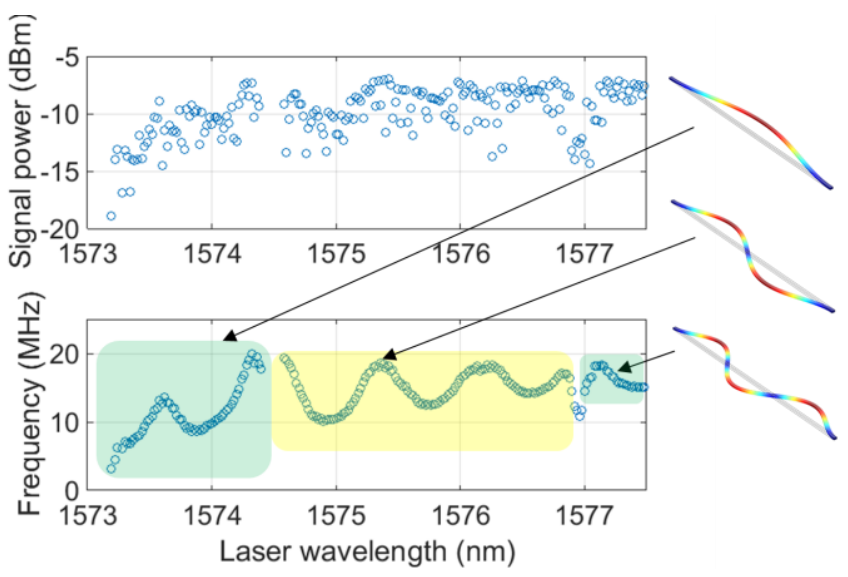

Figure 5. Variation of $R F$ oscillation power and oscillation frequency of the fundamental harmonic with laser wavelength. The laser wavelength is manually tuned from $1573 \mathrm{~nm}$ to $1577.5 \mathrm{~nm}$ and oscillation spectrum is recorded at every wavelength. At $1576.9 \mathrm{~nm}$, the oscillation frequency abruptly drops as a result of the oscillation switching to another mechanical mode. The FEM simulated mode-shapes for the fundamental $n=1, n=2$ and $n=3$ inplane bending modes of the waveguide are shown on the right.

Fig. 6 shows a comparison of three traces recorded at different wavelengths highlighting the vast tuning range. This tuning range is achieved with an input laser power of $14 \mathrm{dBm}$. Accounting for the optical losses for the unoptimized grating couplers ( $8 \mathrm{~dB}$ each at input and output grating couplers), this corresponds to $6 \mathrm{dBm} \approx 4 \mathrm{~mW}$ optical power incident on the device following the input grating coupler. The observed tuning coefficient is thus $525 * 1 \mathrm{e} 4 \mathrm{ppm} / 4 \mathrm{~mW} \approx 1312.5 \mathrm{ppm} / \mu \mathrm{W}$.

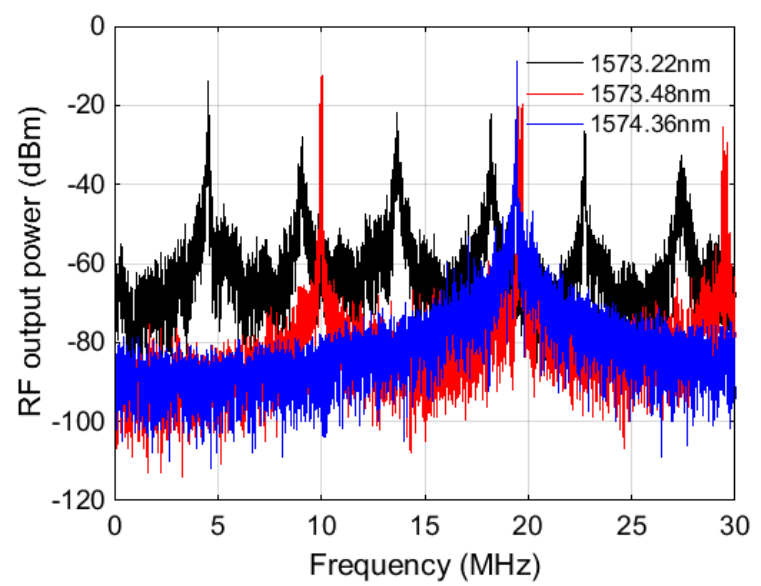

Figure 6. Comparison of RF oscillation spectra at three different wavelengths highlighting the wide tuning range enabled by the optical gradient force. Presence of oscillation harmonics as noticed in this plot, is inherent to non-linear modulation in optomechanical oscillators [8, 13].

As the laser wavelength is varied to $1576.9 \mathrm{~nm}$, the oscillation frequency shows a sharp transition to the $n=2$ in-plane bending mode as shown in Fig. 5. This phenomenon is similar to the transition from 'buckled up' to 'buckled down' mode of operation in nano-mechanical resonators due to opto-mechanical interactions described in [12]. As the laser wavelength is increased further beyond $1577 \mathrm{~nm}$, the oscillations show another sharp transition to the higher frequency $n=3$ mode. 
Non-linear modulation of the laser light on account of the mechanical motion of the resonator results in generation of oscillation harmonics in opto-mechanical oscillators, as previously noted by us and other groups $[8,13]$. The wide tuning range is also noticed for these harmonics. The intensity map shown in Fig. 7 illustrates this observation by plotting out the measured RF power at all frequencies from $100 \mathrm{kHz}$ to $50 \mathrm{MHz}$ as the input laser wavelength is manually varied. The bright regions in this plot correspond to the oscillation signals. The frequency range of tuning is naturally twice as large for the second harmonic as compared to the fundamental oscillation signal for the oscillating mode, as the percentage variation in frequency for all harmonics is constant.

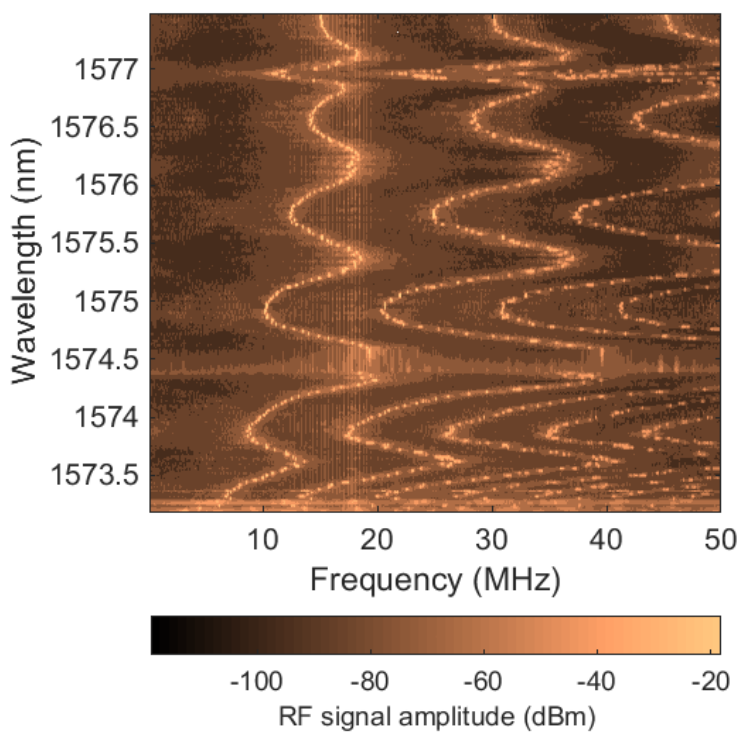

Figure 7. Contour plot showing tuning range for fundamental and higher order harmonics with variation of laser wavelength ( $Y$ axis). The color map represents the $R F$ signal power at every frequency point in the spectrum. The spectrum is recorded from $100 \mathrm{kHz}$ to $50 \mathrm{MHz}$ (X-axis).

\section{CONCLUSION AND FUTURE WORK}

The optical spring effect demonstrated in this paper can be used as a powerful tool to tune the frequency of mechanical oscillators. The tuning coefficient of $1312.5 \mathrm{ppm} / \mu \mathrm{W}$ for the optical spring in this work is significantly larger than $\sim 100 \mathrm{ppm} / \mathrm{V}$ typically achieved by VCXOs. The silicon beam oscillator chosen here is not an ideal choice for reasons well known to the MEMS oscillator community on account of its low effective mass. For low phase noise and frequency stability, choosing a resonator with a high mechanical quality factor and large effective mass is important as it directly translates to low phase noise and low jitter and hence improved frequency stability. Future work could explore adopting this scheme to an opto-mechanical resonator with high mechanical and optical quality factor with an active laser wavelength control loop to demonstrate autonomous sub-ppm operation.

\section{REFERENCES}

[1] J.T.M van Beek, and R. Puers, "A Review of MEMS Oscillators for Frequency Reference and Timing Applications", J. Micromech. Microeng. 22, 013001, 2012.

[2] V. Kaajakari, "Theory and Analysis of MEMS Resonators", Tutorial, Joint Conference of the $65^{\text {th }}$ IEEE IFCS and $25^{\text {th }}$ EFTF, San Francisco, 2011.

[3] "SiTime's MEMS Resonators Outperform Quartz," Press Release, Sunnyvale, 2013. http://www.sitime.com/news/press-releases/451-sitimesmems-resonators-outperform-quartz

[4] S. Sridaran et al., "Low Jitter FBAR based Chip Scale Precision Oscillator," IEEE IUS, pp. 85-88, Chicago, 2014.

[5] R. Ruby et al., "Oven Controlled FBAR Oscillator," IEEE IUS, pp. 1-4, Taipei, 2015.

[6] M. Hossein-Zadeh et al., "Observation of Optical Spring Effect in a Microtoroidal Optomechanical Resonator," Optics Letters, Vol. 32, No. 12, pp. 1611-1613, 2007.

[7] B. S. Sheard et al., "Observation and Characterization of an Optical Spring," Phys. Rev. A, 69, 051801(R), 2004.

[8] S. Tallur et al., "A Monolithic Radiation-Pressure Driven, Low Phase Noise Silicon Nitride Opto-Mechanical Oscillator," Optics Express, 19, pp. 24522-24529, 2011.

[9] J. Rosenberg et al., "Static and Dynamic Wavelength Routing via the Gradient Optical Force," Nature Photonics 3, pp. 478483, 2009.

[10] Q. Lin et al., "Mechanical Oscillation and Cooling Actuated by the Optical Gradient Force," Phys. Rev. Letters, 103, $103601,2009$.

[11] S. Tallur et al., "Electro-Mechanically Induced GHz Rate Optical Frequency Modulation in Silicon," IEEE Photonics Journal 4(5), pp. 1474-1483, 2012.

[12] M. Bagheri et al., "Dynamic Manipulation of NanoMechanical Resonators in the High-Amplitude Regime and Non-Volatile Mechanical Memory Operation," Nature Nanotechnology, 6, pp. 726-732, 2011.

[13] T.O. Rocheleau et al., "Enhancement of Mechanical Q for Low Phase Noise Optomechanical Oscillators," IEEE MEMS, Taipei, pp. 118-121, 2013.

\section{CONTACT}

*S. Tallur, tel: +1-781-937-2151; sgt28@cornell.edu 